\title{
“Te Enseño a Jugar": Caracterización de Movimientos Interaccionales y Formas Lingüísticas Mediante las Cuales se Regula la Interacción Lúdica
}

\section{"I Teach You How to Play": Description of the Interactional Movements and Linguistics Forms Used to Regulate Play Interactions}

\author{
Alejandra Stein y Maia Migdalek \\ Consejo Nacional de Investigaciones Científicas y Técnicas \\ Patricia Sarlé \\ Universidad de Buenos Aires
}

\begin{abstract}
El lenguaje regula la actividad humana, incluyendo el juego; simultáneamente, el juego constituye un medio para el desarrollo lingüístico (Katherine Nelson, 1996; Vigotsky, 1988). El juego resulta un contexto privilegiado para los usos regulativos del lenguaje y para el desarrollo del discurso instructivo (Graves, 1992). Se analiza una muestra intencional conformada por 31 situaciones de juego en las que participaron en el hogar 10 niños de 4 años de barrios marginados de Argentina. El análisis cualitativo (Strauss \& Corbin, 2002) mostró distintos movimientos interaccionales (modelado, preguntas, comparaciones) y formas lingüísticas de regulación de la situación (imperativos, frases verbales de obligación) usados por los adultos, que pueden contribuir al desarrollo del discurso instructivo de los niños.
\end{abstract}

Palabras clave: juego, reglas, regulación, desarrollo lingüístico, discurso instruccional

Language regulates human activity, including play; simultaneously, play is a means for linguistic development (Katherine Nelson, 1996; Vigotsky, 1988). Play situations are a privileged context for the regulative uses of language and for the development of instructional discourse (Graves, 1992). Within this framework, in this paper a purposive sample that includes 31 play situations is analyzed. In these situations participated 10 4-year-old children from marginalized neighbourhoods in Argentina. The qualitative analysis (Strauss \& Corbin, 2002) showed that adults used different interactional movements (modelling, questions, comparisons) alongside with linguistic forms of regulating the situation (imperatives, verbal phrases of obligation), all of which can contribute to the development of instructional discourse in children.

Keywords: play, rules, regulation, linguistic development, instructional discourse

El presente trabajo se enmarca en el área de la relación entre el juego y el desarrollo del lenguaje. Específicamente, se analizan los movimientos interaccionales a través de los cuales

Alejandra Stein y Maia Migdalek, Centro Interdisciplinario de Investigaciones en Psicología Matemática y Experimental, Consejo Nacional de Investigaciones Científicas y Técnicas (CIIPME-CONICET) y Universidad de Buenos Aires, Argentina; Patricia Sarlé, Instituto de Investigaciones en Ciencias de la Educación, Universidad de Buenos Aires, Argentina.

El artículo que se reporta se desarrolla a partir de los resultados de la Tesis Doctoral no publicada de la primera autora, "El entorno de alfabetización en hogares de niños preescolares de sectores urbano-marginados: un estudio de los procesos interaccionales en las situaciones de lectura y escritura", Universidad Nacional de Córdoba, Argentina. De dicha investigación de tesis se ha derivado una serie de estudios actualmente publicados o en prensa en distintas revistas.

La correspondencia relativa a este artículo debe ser dirigida a la Dra. Alejandra Stein, Centro Interdisciplinario de Investigaciones en Psicología Matemática y Experimental, Consejo Nacional de Investigaciones Científicas y Técnicas, Universidad de Buenos Aires, Presidente Perón 2158, 1040, Buenos Aires, Argentina. E-mail: alejandrastein@yahoo.com.ar 
se regulan situaciones de juego con reglas convencionales en hogares de niños de barrios urbanos marginados de Argentina. Estas situaciones pueden constituir una oportunidad para el desarrollo del discurso instruccional.

Desde una perspectiva sociocultural, que otorga un rol primordial al lenguaje y a la interacción social en el desarrollo infantil (Bruner, 1986; Katherine Nelson, 1996; Vigotsky, 1988), se concibe a la interacción como una matriz para la adquisición del lenguaje. Es en el encuentro social en el que se construyen las competencias lingüísticas, a través del proceso dinámico de la interacción, de la gestión de la inter-comprensión, de la negociación del sentido y de posicionamientos recíprocos (Mondada \& Pekarek Doehler, 2001). La interacción tiene lugar en el marco de actividades, por lo que la competencia lingüística también se configura en las contingencias locales de la acción (Pekarek Doehler, 2006). La participación en situaciones compartidas con otros conduce a los niños desde los usos más simples del lenguaje oral a la producción y comprensión de textos orales y escritos progresivamente más complejos (Bruner, 1986; Katherine Nelson, 1996; Vigotsky, 1988).

Diversas investigaciones realizadas en el medio familiar que analizan la interacción madre-niño y su vínculo con el desarrollo lingüístico (e.g., Bruner, 1986; Keith E. Nelson, 1977) mostraron que no es la interacción en sí misma la que influye positivamente en el desarrollo del lenguaje, sino determinados movimientos interaccionales - la expansión y la reestructuración de los enunciados de los niños por parte de las madres- que promueven la comprensión mutua, meta funcional del sistema de interacción (Snow, 1983). Estas estrategias constituyen un ajuste discursivo en el habla adulta y le ofrecen al niño nueva información que este puede procesar en la adyacencia con su propia emisión (Brown \& Bellugi, 1964; Cazden, 1972; Keith E. Nelson, 1977).

Dado que el juego es una de las actividades constitutivas de los primeros años de la vida de los niños, resulta fundamental su consideración en relación a la adquisición del lenguaje. Los trabajos tradicionales de Bruner (1986) han atendido específicamente a esta relación en los primeros juegos entre la madre y el niño. Desde esta perspectiva, se conciben estos juegos tempranos como formatos idealizados, estructuras de acción simples y estandarizadas, que el niño domina paulatinamente. En este marco, el niño puede atender a las formas lingüísticas imbricadas en el juego y desarrollar las primeras formas de la petición y la referencia.

A medida que el niño crece, participa en juegos con formatos diversos y más complejos que requieren que utilice sus recursos lingüísticos para comunicarse con otros, de modo tal que estos comprendan sus intenciones y lleven a cabo sus demandas. El juego pone a los niños en situaciones de interacción social con sus compañeros y los obliga a generar intercambios que justifiquen y coordinen sus acciones con las de otros (Sarlé, 2001). A su vez, el lenguaje constituye uno de los medios centrales para crear y sostener, en la interacción con otros, el argumento del juego y desplegar la secuencia narrativa (Katherine Nelson, 1996; Seidman, Nelson \& Gruendel, 1986). De esta forma, se establece un vínculo bidireccional entre lenguaje y juego (Rosemberg, 2009): el lenguaje permite el desarrollo del juego y el juego, al requerir la puesta en la interacción de recursos lingüísticos y discursivos más complejos, da lugar a que los niños lo desarrollen.

Diversas investigaciones focalizadas en el estudio de las relaciones entre el juego y el lenguaje mostraron que ciertas características de las situaciones lúdicas, particularmente las interacciones entre los participantes, pueden promover el desarrollo del discurso narrativo y el uso de un lenguaje descontextualizado, explícito, con vocabulario preciso y una sintaxis relativamente compleja, como el que caracteriza a la escritura (Dickinson \& Tabors, 2001; Howe, Petrakos, Rinaldi \& LeFebvre, 2005; Manrique \& Rosemberg, 2009; Pellegrini, 1985).

Varios trabajos focalizados en el estudio del habla de los niños en la interacción entre pares durante situaciones de juego sociodramático en el hogar y en el jardín de infantes mostraron que durante el juego sociodramático los niños realizan transformaciones de objetos 
y transformaciones ideacionales, más abstractas e independientes de los objetos presentes. En efecto, el juego dramático, al requerir el cambio de roles de la realidad a la fantasía, se sostiene a través de transformaciones verbales explícitas que evitan la ambigüedad. Los niños deben recurrir a formas en las que priman las referencias endofóricas - los elementos se nombran, definen y caracterizan dentro del texto- Asimismo, requieren la definición de los objetos y de los personajes por medio de frases nominales complejas que incluyen modificadores directos e indirectos. Para definir estas transformaciones con sus compañeros de juego, evitando ambigüedades, los niños deben emplear un lenguaje explícito, descontextualizado y con una sintaxis relativamente compleja (Howe et al., 2005; Pellegrini, 1985).

También se ha visto que el juego sociodramático promueve el desarrollo del discurso narrativo, en tanto que en la situación de juego los niños representan eventos de la vida cotidiana y para ello deben analizar y reconstruir la estructura temporal y causal de estos eventos, estructura similar a la estructura de las narraciones (Pellegrini, 1985; Rosemberg, 2009).

Como se desprende de las investigaciones mencionadas, la mayoría de los estudios previos se focalizó en el análisis de situaciones de juego sociodramático y sus relaciones con el desarrollo de un estilo de lenguaje escrito y del discurso narrativo. La mayoría de estos estudios fue realizada con población de nivel socioeconómico medio. Son escasas las investigaciones realizadas en poblaciones urbanas marginadas (Manrique \& Rosemberg, 2009) y pocos los trabajos que han atendido a la relación entre los juegos con reglas convencionales y el desarrollo del lenguaje y, específicamente, del discurso instruccional.

\section{Juegos con Reglas Convencionales: Reglas, Regulación de la Situación Lúdica y Discurso Instruccional}

Desde el enfoque sociocultural (Vigotsky, 1988) se ha señalado que el origen de la regulación de la propia actividad tiene lugar en la interacción madre-niño. La madre, mediante instrucciones, guía y organiza la atención del niño. Luego, el pequeño comienza a darse órdenes a sí mismo en forma de lenguaje externo y posteriormente como lenguaje interno. De este modo, el origen del acto voluntario tiene lugar primero en el plano interpsicológico y más tarde en el plano intrapsíquico de la autorregulación, para, finalmente, interiorizarse como pensamiento. La regulación de una actividad específica — el control de la misma - implica el conocimiento de lo que ocurrirá para poder dominar los distintos aspectos involucrados en dicha actividad (Bruner, 1986). En los juegos la regulación de la situación y de la propia acción se ve condicionada por las reglas que el juego impone, a las que el niño debe someterse para jugar (Linaza \& Maldonado, 1987; Vigotsky, 1988). Frente al impulso de lo que le gustaría hacer, el niño se encuentra con la limitación que el juego mismo le impone. Esta limitación varía según el tipo de juego. En los juegos sociodramáticos las reglas están contenidas en los papeles sociales y las acciones que producen los jugadores y se refieren a la situación imaginada puesta en acto. Estas reglas dan sentido a la acción y le otorgan claves de significado. Son reglas que no se deciden por adelantado, sino que se desprenden de las características de la situación a representar. Distintas investigaciones han mostrado que los juegos sociodramáticos se apoyan en los guiones que los niños construyen a partir de su participación en las situaciones sociales mediadas por los adultos (Katherine Nelson, 1996; Sarlé, 2001; Seidman et al., 1986). En los juegos con reglas convencionales, en cambio, las reglas vienen dadas arbitrariamente por el juego mismo: es el seguimiento de la regla lo que otorga sentido al juego y lo determina. Cambiar las reglas supone un cambio en el juego (Garrido, 2008; Sarlé, 2001).

Desde el punto de vista lingüístico, el reglamento de un juego está organizado en una secuencia instruccional cuya finalidad es hacer actuar al destinatario (Bronckart, 2004; Silvestri, 1995). En este sentido, Silvestri (1995) afirma que el discurso instruccional es un caso especial entre los tipos discursivos, dado que su vínculo con la actividad es el más explícito y directo. $\mathrm{Su}$ función es la enseñanza de una serie de pasos ordenados que conlleva un plan de acciones 
jerarquizadas y requiere, por lo tanto, de un emisor experto en dicho procedimiento (Fajre \& Arancibia, 2000; Silvestri, 1995). A medida que el destinatario adquiere la competencia para actuar según los pasos señalados por la instrucción, ella desaparece como texto, quedando internalizada como práctica (Fajre \& Arancibia, 2000).

Los enunciados que configuran el discurso instruccional constituyen una forma de enunciados directivos, cuya finalidad es regular la acción del destinatario. La orden es la forma prototípica del acto de habla (Searle, 1986), por la cual se transmiten explícitamente las directivas, y la forma gramatical del español congruente con ella es el modo imperativo (Silvestri, 1995).

Las actividades lúdicas, específicamente aquellos juegos con reglas convencionales, resultan un contexto privilegiado para los usos regulativos del lenguaje y, por lo tanto, para el desarrollo del discurso instruccional (Graves, 1992; Jaimes Carvajal \& Rodríguez Luna, 1996). Tener que explicar las reglas a otros puede dar lugar a que los participantes empleen usos de lenguaje más explícitos, generando oportunidades para que los niños pequeños los aprendan.

Los propósitos del presente estudio se enmarcaron en los objetivos específicos de una investigación más amplia (señalada en la Nota de las Autoras), focalizada en el estudio de las características del entorno de alfabetización en hogares de niños de sectores urbano marginados de Argentina, atendiendo, en particular, a los procesos interaccionales que promueven la alfabetización temprana de estos niños.

De la investigación marco mencionada se han desarrollado otros estudios basados en la misma muestra, pero con diferentes objetivos y analizando distintos datos. Así, se estudiaron las redes de colaboración que se establecían entre los participantes durante la consecución de las distintas actividades de alfabetización (Stein \& Rosemberg, en prensa a), los patrones y las modalidades de interacción en situaciones sucesivas de alfabetización familiar (Stein \& Rosemberg, en prensa b), las intervenciones de los adultos y de los niños mayores que interactúan con los pequeños durante situaciones de lectura de cuentos (Stein \& Rosemberg, en prensa c) y durante actividades focalizadas en el aprendizaje del sistema de escritura — juegos con sonidos y letras, establecimiento de correspondencias grafema-fonema, escritura de palabras- (Stein \& Rosemberg, 2011).

El presente trabajo buscó aportar al conocimiento de los procesos de interacción en las situaciones de alfabetización familiar en las que tienen la oportunidad de participar niños de poblaciones urbanas marginadas de Argentina. Específicamente, el objetivo consistió en analizar las matrices interaccionales en situaciones de juego con instrucciones, en hogares de niños de cuatro años de barrios urbanos marginados de las ciudades de Buenos Aires y Córdoba, Argentina, con el propósito de identificar y caracterizar los movimientos interaccionales y las formas lingüísticas mediante los cuales los adultos y los niños mayores transmiten las reglas de juego y regulan la situación lúdica con los pequeños, que permiten que el juego tenga lugar, sostienen la interacción del niño y facilitan el desarrollo del discurso instructivo por parte de los pequeños.

\section{Método}

El presente estudio se enmarcó en las perspectivas interpretativas de la investigación científica que enfatizan la comprensión del objeto de estudio en su carácter contextualizado y en el análisis cualitativo (Forni, 1993). Las investigaciones que responden a esta lógica se basan en la generación de categorías conceptuales, con sus propiedades y relaciones mutuas, y en la comparación de constructos e hipótesis generados a partir de fenómenos observados en diferentes situaciones (Strauss \& Corbin, 2002).

El modo de operar eminentemente cualitativo implicó la necesidad de elaborar estrategias diversas que pudieran dar cuenta de la complejidad del fenómeno en estudio. Se elaboró un di- 
seño con el fin de construir evidencia empírica sobre las interacciones durante el juego con instrucciones. Consistió en la observación de situaciones inducidas por medio de la introducción en los hogares de materiales de alfabetización temprana que incluían juegos con instrucciones en el marco del Programa Oscarcito (Rosemberg \& Borzone, 2004a), un programa de desarrollo lingüístico y cognitivo infantil elaborado como transferencia de resultados del proyecto El desarrollo lingüistico y cognitivo en la primera infancia: un estudio psicolingüistico y sociocultural en barrios urbano marginales de Buenos Aires (Rosemberg \& Borzone, 2004b). El objetivo del programa es la promoción del desarrollo lingüístico y cognitivo y la alfabetización temprana en niños de tres a cinco años que viven en barrios de extrema pobreza. A través del programa se llevan a cabo acciones educativas en los hogares de los niños y en los jardines de infantes a los que asisten. Estas acciones recuperan los conocimientos y el lenguaje de los niños y buscan, a partir de allí, ampliar su vocabulario, su conocimiento del mundo, sus estrategias de discurso, el aprendizaje de la variedad lingüística estándar y el ingreso al proceso de alfabetización.

\section{Las Situaciones Analizadas}

Las situaciones lúdicas analizadas son parte de los datos recogidos en el marco de la investigación más amplia mencionada. Las situaciones de juego que conforman el corpus de este estudio fueron inducidas a partir de la implementación del Programa Oscarcito, en el que las familias de los niños participan en talleres de capacitación sobre desarrollo infantil y reciben una serie de 12 libros socioculturales (serie "En la casa de Oscarcito") destinados a facilitar a los niños el acceso a la alfabetización en el contexto de sus hogares. Cada volumen trae una hoja recortable en la que se presentan distintos juegos (memotest, dominó, lotería, entre otros) para que los niños jueguen con sus familias. La presentación de las reglas de los juegos en los libros no es uniforme: mientras que algunos juegos no presentan ninguna indicación sobre cómo jugar, otros presentan un reglamento completo. En todos los casos las reglas son explicadas oralmente por la coordinadora del encuentro y se dedican unos minutos de la reunión para que dos jugadores jueguen, a modo de ejemplo.

El conjunto de datos obtenidos en la investigación más amplia mencionada consistió en 124 situaciones de alfabetización registradas en los hogares de 30 niños de cuatro años de edad. En estas situaciones los niños realizaron junto con sus familiares 617 actividades de lectura de cuentos, escritura de palabras, juegos con los sonidos de las palabras y juegos con reglas convencionales. Se realizaron en promedio 6,5 observaciones en cada hogar —entre 5 y $11 \mathrm{ob}$ servaciones-, una vez por mes, durante el período de implementación del programa (12 meses aproximadamente).

En un artículo anterior Stein y Rosemberg (2011) analizaron las actividades focalizadas en el desarrollo de conocimientos y habilidades referidos al sistema de escritura, esto es, 84 actividades de escritura — de palabras (37), de frases (7) - y 216 actividades focalizadas en el desarrollo de habilidades fonológicas (juegos con sonidos y letras, establecimiento de correspondencias grafema-fonema).

El análisis del estudio reportado en este artículo se centró en las modalidades de intervención de los adultos durante las actividades mencionadas que pueden promover oportunidades para aprendizaje de la escritura (Stein \& Rosemberg, 2011). Se identificaron y analizaron las 31 actividades de juego - memotest, dominó, lotería, aparear número y cantidad, rompecabezas y recorrido- con reglas convencionales registradas.

\section{Participantes}

Participaron 10 niños de cuatro años de edad (dos de Córdoba y ocho de Buenos Aires), junto con sus padres, hermanos y otros familiares que interactuaban con ellos. Estos niños, que formaban parte de los 30 participantes de la investigación más amplia, fueron seleccionados debido a que en sus hogares tuvieron lugar las 31 situaciones de juego analizadas. 


\section{Instrumento}

Los datos se recogieron mediante observaciones audiograbadas de la interacción verbal. También se tomaron registros escritos acerca de otros rasgos de las situaciones de juego: disposición espacial de los participantes e interacciones kinésicas y gestuales, entre otros.

A los participantes — adultos y niños mayores- se les solicitó que realizaran de modo habitual las actividades incluidas en los libros. El observador respondía los comentarios del niño y de sus familiares, pero no promovía conversaciones ni actividades específicas.

No se pautó previamente la duración de la visita; las observaciones se extendieron durante el tiempo que duró la situación de lectura y la realización de las actividades incluidas en los libros.

\section{Procedimiento}

Se solicitó a las familias la participación voluntaria en el estudio. Respecto de los resguardos éticos, se aplicaron los procedimientos recomendados por las asociaciones profesionales internacionales en relación al trabajo y observación con niños, los principios establecidos por la Convención Internacional sobre los Derechos del Niño y lo establecido en la Ley $\mathrm{N}^{\mathrm{o}} 114$ de Protección Integral de los Derechos de Niños, Niñas y Adolescentes de la Ciudad de Buenos Aires. Es decir, se informó a los adultos responsables de los detalles y requerimientos del estudio y se les solicitó autorizar la participación de los niños a su cargo en las diferentes actividades (situaciones de juego propuestas por el cuadernillo previamente entregado). Junto con ellos se organizó la agenda de trabajo de campo. Estos procedimientos son los aprobados por el Comité de Ética institucional (CONICET-CIIPME), que corrobora el cumplimiento de los principios éticos pertinentes.

\section{Análisis de la Información}

Las situaciones de juego fueron analizadas empleando un procedimiento cualitativo que combina la elaboración inductiva de categorías, en base al método comparativo constante (Strauss \& Corbin, 2002), y su consideración, atendiendo a otros marcos conceptuales, como los propuestos por el análisis de la conversación (Goodwin, 2000; Sacks, Schegloff \& Jefferson, 1974) y el microanálisis discursivo de la interacción (Gumperz, 1984).

Se generaron categorías para dar cuenta de las interacciones entre los participantes destinadas a regular las propias acciones y las de los demás en la situación lúdica. En el análisis inductivo se identificaron y conceptualizaron en los registros de observación aquellos aspectos de los movimientos conversacionales que los participantes realizaban en la situación para explicar el juego a los niños pequeños. Siguiendo el procedimiento de comparación constante, una vez categorizados los aspectos más relevantes de los movimientos conversacionales, se realizó un análisis comparativo de los incidentes comprendidos por cada una de las categorías. Este análisis permitió identificar las propiedades de cada una de ellas y delimitar un sistema conceptual que da cuenta de la regulación de las situaciones lúdicas en entornos familiares.

Atendiendo a las propuestas del análisis conversacional (Goodwin, 2000), en el proceso de elaboración de categorías y de propiedades se atendió a la información lingüística y a un conglomerado de información no lingüística: gestos, miradas, posicionamiento corporal e información del contexto situacional. Esta información no lingüística se yuxtaponía a la información lingüística en la configuración de los movimientos (Sacks et al., 1974) que los participantes realizaban para explicar las reglas del juego y jugar. 
El sistema de categorías y propiedades elaborado atendió a los siguientes aspectos:

1. Quiénes participan en las situaciones de juego: (a) madre, (b) niño/s mayor/es, (c) niño pequeño.

2. Quiénes regulan la situación lúdica: (a) madre, (b) niño mayor, (c) madre y niño mayor.

3. Los movimientos interaccionales — que incluyen componentes verbales y no verbales- por medio de los cuales se lleva a cabo dicha regulación: (a) modelado, (b) formulación de preguntas, (c) establecimiento de comparaciones entre juegos, (d) órdenes que seguían el procedimiento pautado por la regla del juego.

4. Si la regulación incluye o no la explicación de las reglas del juego: (a) se regula solo la alternancia de turnos y la realización de una acción concreta, (b) la regulación incluye explicar la regla que subyace al juego.

5. El momento en que las reglas son explicadas: (a) antes, (b) durante el juego.

6. Las características de las formas lingüísticas destinadas a regular las propias acciones y las de los demás durante el juego: (a) más explícitas en su directividad y más contextualizadas, (b) menos explícitas en su directividad y más descontextualizadas.

Este análisis fue realizado conjuntamente por las autoras, cada una de las cuales codificó luego independientemente el 20\% de los casos. En un 90,3\% de los casos acordaron en los códigos asignados. Luego de discutir y acordar criterios para categorizar los casos periféricos, el $40 \%$ restante de las situaciones fue codificado por la primera autora.

Asimismo, se cuantificaron algunos de los aspectos analizados con el objetivo de conocer cómo estos aspectos se distribuyen en el corpus en estudio.

\section{Resultados}

\section{Los Movimientos Interaccionales a Través de los Cuales se Regula la Situación Lúdica}

Uno de los primeros aspectos analizados fue quién regulaba la situación de juego, regulación que implica el conocimiento de los distintos aspectos que la actividad involucra, entre ellos, la regla del juego. Se observó que las situaciones lúdicas estaban reguladas por la madre, un hermano mayor y la madre y el niño mayor conjuntamente. Algunos juegos, tales como el memotest y el dominó, no eran conocidos mayormente por las madres. En estos casos eran los hermanos mayores quienes asumían principalmente la regulación de la actividad y explicaban las reglas.

El análisis se focalizó luego en la identificación de los movimientos interaccionales verbales y no verbales por medio de los cuales los adultos y niños mayores regulaban la actividad de los pequeños. Los resultados mostraron que los adultos y los niños mayores regulaban la situación lúdica por medio de: el modelado, la formulación de preguntas con diferentes funciones pragmáticas, el establecimiento de comparaciones con otros juegos conocidos y el empleo de órdenes que seguían el procedimiento pautado por la regla del juego.

Asimismo, resultó pertinente atender al momento en el que los adultos y los niños mayores transmitían las reglas a los pequeños. El análisis evidenció que, en ocasiones, los adultos y los niños mayores explicaban las reglas del juego antes del inicio de la actividad; en otras oportunidades las reglas se explicaban durante el juego. En la mayoría de los casos la explicación de las reglas estaba acompañada por el modelado de las mismas. En la Tabla 1 se presentan los porcentajes de situaciones en las que las explicaciones se producían antes o durante el juego y con o sin modelado. 
Tabla 1

Explicación de las Reglas en las Actividades de Juego: Antes o Durante y con o sin Modelado (\%)

\begin{tabular}{lccc}
\hline & Modelado & Sin modelado & Total \\
\hline Antes del juego & 17,9 & 7,1 & 25,0 \\
Durante el juego & 50,0 & 25,0 & 75,0 \\
Total & 67,9 & 32,1 & $100,0(31)$ \\
\hline
\end{tabular}

En la mayoría de los casos (75\%) los adultos y niños mayores explicaban las reglas jugando. En el marco de la explicación durante el juego los adultos y niños mayores modelaban la actividad (50\%) sin explicitar la finalidad del juego y, en ocasiones, incorporaban al pequeño en el modelado, como se observa en el siguiente fragmento de intercambio en el que participan Zoe (la niña), su madre y su hermana Joseline de seis años:

[El juego consiste en aparear una serie de tarjetas que contienen ilustraciones de bichos con el número correspondiente según la cantidad de bichos en cada tarjeta. Joseline explica el juego].

Joseline: Cada una tiene que tener sus numeritos. Yo tengo seis y me lo agarro [toma una tarjeta con seis bichos y otra tarjeta con el número 6]. Yo me llevo este, ¿cuál te llevás vos?

Madre: Yo miro y aprendo, asi después jugamos.

Zoe: Mirá. Este es mío y este es tuyo. El tres [toma una tarjeta que tiene el dibujo de tres bichos].

Joseline: ¿Adónde hay tres?

Zoe: Mirá.

Joseline: De estas [señala varias tarjetas], ¿cuál es tuya?

Zoe: Estas tres. No, esta sola [toma la tarjeta que tiene el número 3].

En el intercambio Joseline le explica a su hermana cómo jugar realizando las acciones de acuerdo con las reglas, al tiempo que verbaliza esas mismas acciones: Cada una tiene que tener sus numeritos. Yo tengo seis y me lo agarro. No se explicita que la finalidad del juego es reunir los números con los dibujos correspondientes. Se pone el acento en el aspecto técnico de la regla que permite la participación en el juego más que en aquellos componentes que están vinculados con saber quién gana o quién pierde, aspectos relacionados con el término de la actividad.

Muchas de las preguntas que formula Joseline no tienen la función de obtener información por ella desconocida. Se trata de preguntas que buscan controlar el conocimiento que su hermana posee de la regla y focalizar la atención de la pequeña en la secuencia de pasos requerida para jugar. En muchas de las situaciones analizadas se observó que los adultos y niños mayores regulaban la actividad por medio de la formulación de preguntas referidas implícitamente a la finalidad del juego, para proporcionar al niño un andamiaje que le permitiera internalizar las reglas. De esta manera, el niño puede desempeñarse en su turno sin que su compañero de juego explícitamente le diga lo que debe hacer. En el intercambio la pregunta de Joseline - ¿cuál te llevás vos? - tiene por objetivo que su hermana elija y tome una tarjeta, primer paso dentro del turno de juego; en el segundo caso — ¿Adónde hay tres?_, es una pregunta de información conocida por Joseline y apunta a la finalidad del juego: identificar una tarjeta que pueda aparearse con la elegida previamente en función de la cantidad.

En el siguiente fragmento de intercambio la madre de Agustín enuncia las reglas al inicio del juego, pero vuelve a explicarlas en su turno, verbalizando los procesos mentales que se deben realizar para participar de la actividad de acuerdo a las reglas:

[Agustín y su madre recortan las fichas del memotest y luego juegan].

Madre: Este juego es para acordarse las figuritas ino? Cuando sale una figurita, tenés que levantar otra figurita igual. Si no son iguale[s], hay que darla vuelta de nuevo así [da vuelta una ficha y la coloca boca abajo, sin que se vea el dibujo] y si te acordás otra vuelta, entonces... 
Agustín: Ganás.

Madre: Ganás. Despué[s] hay que mezclarlas un poco.

Agustín: Sí.

[Terminan de recortar todas las fichas y las acomodan sobre la mesa, con la ilustración boca abajo].

Madre: ¿Y ahora? ¿Quién es primero?

Agustín: Yo.

Madre: ¿Vo[s]? Bueno, a ver, levantá una.

Agustín: [Da vuelta una ficha que tiene el dibujo de un submarino].

Madre: Uy, un submarino. Tenés que buscar el otro submarino, a ver.

Agustín: [Da vuelta otra ficha. El dibujo no es un submarino].

Madre: ;Ah, no es!

Agustín: Te toca a vo[s].

Madre: Ahora me toca a mí. A ver yo... que yo no me acuerdo. A ver [da vuelta una ficha y la mira]. iUn tren!

¿Dónde estará el otro tren? A ver, ¿acá? [Señala una ficha]

Agustín: No sé.

Madre: Vamos a ver [da vuelta otra ficha]. Nooo.

Agustín: Pediste [perdiste]. Ahoda [ahora] a mí.

Madre: Un cohete [nombra la ficha obtenida por su hijo].

Agustín: ¿Dónde estaba?

Madre: No sé.

Agustín: Acá [da vuelta otra ficha; es otro cohete].

La primera pregunta de la madre de Agustín - ¿Y ahora? ¿Quién es primero?_- está destinada a la regulación de los turnos del juego; Agustín puede ocupar el primer turno gracias a la regulación de su madre, quien lo guía mediante órdenes: Bueno, a ver, levantá una; tenés que buscar el otro submarino, a ver. Luego, en su turno, la madre verbaliza los procesos mentales que son necesarios para jugar de acuerdo a las reglas, mediante un enunciado negativo y una pregunta que recupera la finalidad del juego, recordar la ubicación de las fichas y buscar las iguales: Yo no me acuerdo... ¿Dónde estará el otro tren? A ver, ¿acá?

En ocasiones los adultos recuperan conocimientos compartidos con el pequeño y establecen comparaciones entre el juego en desarrollo y otros juegos conocidos, como Denis y su madre:

[Denis y su madre juegan al dominó].

Madre: ¿Cómo es esto, mami? [Se dirige a la observadora].

Observadora: Primero hay que recortarlo. Después se reparten las fichas.

Madre: Es como las cartas, Deni.

La madre activa conocimientos previos del niño que le facilitarán al pequeño el acceso al nuevo juego y el dominio de sus reglas.

Asimismo, se observó que los adultos y los niños mayores regulan la situación lúdica realizando las acciones requeridas por el juego en el turno correspondiente al niño. Esto es lo que sucede en el siguiente fragmento de intercambio, en el que Denis (el niño) y su hermano Franco de 11 años juegan a un juego de recorrido, usando un dado y un tablero:

Franco: Yo primero [tira el dado y cuenta los casilleros que avanza] Uno, dos, tres, cuatro, cinco y seis. Ahora vos tirá. En otro lado, acá tirá.

Denis: [Tira el dado].

Franco: A ver, cinco. Uno, dos, tres, cuatro, cinco [cuenta los casilleros y mueve la ficha de Denis]. Ahora yo tiro.

En el intercambio el hermano mayor de Denis simplifica la actividad: regula mediante órdenes las acciones más sencillas que el niño puede realizar — tirar el dado-y realiza aquellas que considera más difíciles para el pequeño — contar y mover la ficha en el tablero-.

La regulación de la situación lúdica a través de los distintos movimientos interaccionales era llevada a cabo mayoritariamente por un adulto o niño mayor. También se identificaron casos en los que en la misma situación se registró cierto traspaso del control del juego del adulto o del niño mayor hacia el niño pequeño: en el intercambio entre Agustín y su madre ella verbaliza los procesos mentales que debe realizar para participar del juego - ¿Dónde estará el otro tren?-. Posteriormente, en su turno, Agustín repite la misma pregunta — ¿Dónde estaba?-, regulando, de este modo, su propia acción. 


\section{Caracterización de las Formas Lingüísticas Empleadas en la Regulación de la Situación Lúdica}

Como segunda instancia de análisis se atendió a las formas lingüísticas empleadas por los participantes destinadas a regular las propias acciones y las de los demás durante el juego. El análisis se focalizó en los movimientos interaccionales de modelado y órdenes, teniendo en cuenta el estrecho vínculo lingüístico de este tipo de movimientos interaccionales y el discurso instruccional.

El análisis mostró que para regular la actividad conjunta durante el juego los adultos y los niños mayores, al formular las órdenes, eligen formas de imperativo y modalizaciones con la frase verbal de obligación tener + que + infinitivo en segunda persona, como sucede en el siguiente intercambio:

[Ovidio, su madre y su hermana de 8 años juegan al memotest].

Ovidio: [Toma una ficha].

Madre: No, ahí no estaba; ese es el cohete. ¿Cuál submarino sacaste primero? Ese no era.

Ovidio: [Da vuelta varias fichas].

Hermana: No lo tenés que ver.

Madre: Dale, sacá otro. Sacá otro, igual a ese [señala la ficha con el dibujo del submarino].

Los imperativos - sacá - y las formas modalizadas por la frase verbal de obligación en segunda persona — tenés que ver - son formas explícitas en su directividad y contextualizadas, esto es, formas conjugadas que interpelan al niño presente en la situación.

Cuando el adulto o el niño mayor modelizaba para mostrar cómo jugar se registraron formas verbales en primera persona, como en el siguiente ejemplo:

[Pablo y su madre juegan al dominó].

Madre: Acá, mirá. Yo tengo un perrito aquí, Pablo. ¡Yo voy a poner a acá porque tengo un perrito!

Ahí al lado del perrito voy a poner. Ahora si vos tenés en tu carta... Ay, ¿qué es esto? [señala una ficha que está sobre la mesa]

Pablo: ¡Tengo un elefante!

Madre: Acá, mirá.

Todas las formas antes mencionadas -imperativo, modalizaciones con tener $+q u e+$ infinitivo en segunda persona, formas verbales en primera persona- contrastan con otras formas lingüísticas identificadas en las situaciones con menor frecuencia: formas pasivas con el pronombre se y frases verbales de obligación impersonales haber + que + infinitivo. Estas formas son menos explícitas en su directividad, ya que no remiten en forma directa a los participantes de la interacción; señalan la generalidad del procedimiento requerido para participar en un determinado juego e implican un grado mayor de abstracción. Las formas pasivas se utilizan para regular la actividad global y no como medio de regulación de un acto individual preciso, como se observa en el siguiente intercambio en el que Zoe, su madre y su hermana Joseline de seis años juegan al memotest:

Madre: Mirá cómo se juega, Joseline.

Zoe: Asi no se juega [se dirige a Joseline que sigue dando vuelta fichas].

Madre: Tenés que buscar el mismo dibujo.

También se observó el empleo de las formas pasivas con el pronombre se, haciendo referencia a los pasos necesarios para jugar:

[Patricia, su madre y su hermana Aylén de cinco años juegan a la lotería].

Madre: Lotería dice: [lee las instrucciones que acompañan al juego] "Cada jugada se coloca en un cartón de fichas, se coloca en una bolsa o sobre la mesa y saca la ficha de a una y se muestra para que todos sepan que salió. El que tiene en su cartón el mismo dibujo que salió en la ficha se pone una pelotita”.

[Candela y su madre juegan un juego de recorrido].

Madre: Yo... así se tira [tira el dado], yo te digo así, hacé así y tirá el dado. Tirá ahí arriba, ¿̇a ver? [agita el dado en la mano]. 
En el fragmento del juego de Patricia, si bien la madre de la niña emplea la forma pasiva para regular actos individuales precisos — se coloca, se muestra-, está leyendo las instrucciones escritas que acompañan al juego. En el ejemplo del juego de Candela la forma pasiva es reformulada en su contexto inmediato en un imperativo - así se tira, yo te digo así, hacé así y tirá el dado-.

\section{Discusión}

El presente trabajo, si bien tomó como objeto de estudio un número limitado de situaciones de juego, su análisis posibilitó la elaboración de categorías conceptuales que dan cuenta de los modos en los que se regulan las interacciones lúdicas que tienen lugar en hogares urbanos marginados. El análisis permitió identificar los movimientos interaccionales que los adultos y los niños mayores llevan a cabo para regular la situación lúdica con los pequeños: el modelado, la formulación de preguntas con diferentes funciones pragmáticas, el establecimiento de comparaciones con otros juegos conocidos y el empleo de directivas. En las situaciones analizadas se observó que los movimientos interaccionales de los adultos y niños mayores permiten que el juego tenga lugar y sostienen la participación del niño.

Si bien se observó que la regulación de las acciones de los niños suele ser externa a ellos - es llevada a cabo por la madre o niños mayores-, también se registraron casos en los que parece haber un traspaso de la regulación de la actividad así como una internalización de las reglas del juego por parte de los pequeños. En algunos de los casos observados el niño regula su propia acción por medio de intervenciones que adoptan formas lingüísticas similares a aquellas empleadas por los otros participantes. Ello concuerda con los trabajos que plantean una relación bidireccional entre lenguaje y juego (Bruner, 1986; Rosemberg, 2009): el lenguaje posibilita el traspaso del control del juego, en tanto el niño puede comenzar a regular la situación lúdica a partir de la interacción con su madre o niños mayores.

A su vez, el traspaso del control del juego al niño adquiere especial relevancia si se considera que el desarrollo del lenguaje implica un aprendizaje de tipo procedural (Katherine Nelson, 1996; Rosemberg, 2009). Las estructuras y formas lingüísticas complejas se adquieren y desarrollan en situaciones en las que el lenguaje se usa con propósitos diversos. De este modo, el traspaso del control del juego puede contribuir, no solo al desarrollo de habilidades de regulación de la propia conducta y de la conducta de otros, sino también al desarrollo del discurso instructivo por parte de los niños pequeños; los niños entran en contacto con el discurso instruccional. Para contribuir a la regulación de la situación lúdica (Silvestri, 1995) los distintos participantes actualizan los recursos lingüísticos que poseen y recurren a diversas formas lingüísticas que pueden considerarse precursores de formas más elaboradas de discurso instruccional, discurso fundamental para la comprensión de consignas en el ámbito escolar (Linaza \& Maldonado, 1987; Vigotsky, 1988).

Los resultados del presente estudio mostraron, asimismo, que la explicación de las reglas suele ocurrir durante el desarrollo de la situación lúdica y el énfasis está puesto en el aspecto instrumental o técnico de la regla. De modo consistente con este hecho, las formas lingüísticas preferidas para regular el juego son los imperativos y la frase verbal de obligación en segunda persona, formas explícitas en su directividad y contextualizadas, esto es, formas conjugadas que interpelan al niño.

Aunque en las situaciones de juego analizadas se observó el predominio de un lenguaje contextualizado, también se identificaron casos en los que, al igual que en las situaciones de juego sociodramático estudiadas en otras investigaciones (Howe et al., 2005; Pellegrini, 1982, 1985), para regular y llevar adelante la situación lúdica, los adultos y niños mayores que interactúan con los pequeños recurren a un estilo de lenguaje más descontextualizado, empleando formas menos explícitas en su directividad: formas pasivas con el pronombre se y frases verbales de obligación impersonales. 
Esta forma de discurso explícito, en la que el lenguaje proporciona toda la información relevante para la comprensión entre los interlocutores (Chafe, 1985), es característica de los textos escritos. Como ha sido señalado en diversas investigaciones, el dominio temprano de formas de lenguaje descontextualizado resulta importante para el acceso a la alfabetización (Simons \& Murphy, 1988; Snow, 1983; Snow, Porche, Tabors \& Harris, 2007).

La habilidad discursiva de los niños para comprender y producir textos que presentan este estilo de lenguaje, caracterizado por estructuras sintácticas más complejas, puede desarrollarse en los intercambios orales en los que los niños tienen la oportunidad de participar, tales como las situaciones compartidas de juegos con instrucciones, y se transfiere y capitaliza luego en las interacciones que el niño tiene que realizar con la escritura. Constituyen, en este sentido, una preparación para la alfabetización (Snow, 1983; Snow et al., 2007; Watson, 1996), la que ha mostrado también una importante asociación con el éxito escolar (Michaels, 1988; Simons \& Murphy, 1988).

Cabe señalar que el conocimiento de las formas de regulación de las situaciones de juego con instrucciones puede constituir un insumo para el diseño e implementación de intervenciones educativas destinadas a promover el desarrollo del discurso instruccional en niños pequeños. Para ello, los resultados del presente estudio, limitados a un número reducido de niños de población urbana marginada, podrían ser la base para una investigación futura de diseño comparativo, en la que se atiendan a las similitudes y diferencias en las formas de regulación de las situaciones de juego, tanto dentro como entre poblaciones de distinta procedencia sociocultural.

\section{Referencias}

Bronckart, J. (2004). Actividad verbal, textos y discursos. Madrid, España: Fundación Infancia y Aprendizaje.

Brown, R. \& Bellugi, U. (1964). Three processes in the child's acquisition of syntax. Harvard Educational Review, 34, 133-151.

Bruner, J. (1986). El habla del niño. Barcelona, España: Paidós.

Cazden, C. B. (1972). Child language and education. New York, NY: Holt, Rinehart \& Winston.

Chafe, W. L. (1985). Linguistic differences produced by differences between speaking and writing. En D. R. Olson, N. Torrance \& A. Hildyard (Eds.), Literacy, language and learning: The nature and consequences of reading and writing (pp. 105-123). New York, NY: Cambridge University Press.

Dickinson, D. K. \& Tabors, P. O. (2001). Beginning literacy with language: Young children learning at home and school. Baltimore, MD: Paul H. Brookes.

Fajre, C. \& Arancibia, V. H. (2000). La consigna: un manual de instrucciones para leer la escuela. Didáctica (Lengua y Literatura), 12, 121-138.

Forni, F. (1993). Estrategias de recolección y estrategias de análisis en la investigación social. En F. Forni, M. Gallard \& I. Vasilachis de Gialdino (Eds.), Métodos cualitativos II. La práctica de la investigación (pp. 23-64). Buenos Aires, Argentina: Centro Editor de América Latina.

Garrido, R. (2008). Juegos con reglas y números. En P. Sarlé, (Coord.), Enseñar en clave de juego: enlazando juegos y contenidos (pp. 103-133). Buenos Aires, Argentina: Novedades Educativas.

Goodwin, C. (2000). Action and embodiment within situated human interaction. Journal of Pragmatics, 32, 1489-1522. doi:10.1016/S0378-2166(99)00096-X

Graves, D. (1992). Exploraciones en clase: los discursos de la no ficción. Buenos Aires, Argentina: Aique.

Gumperz, J. (1984). Communicative competence revisited. En D. Schiffrin (Ed.), Meaning, form and use in context: Linguistic applications (pp. 278-289). Washington, DC: Georgetown University Press.

Howe, N. Petrakos, H., Rinaldi, C. M. \& LeFebvre, R. (2005). “This is a bad dog, you know...”: Constructing shared meanings during sibling pretend play. Child Development, 76, 783-794. doi:10.1111/j.1467-8624.2005.00877.x

Jaimes Carvajal, G. \& Rodríguez Luna, M. E. (1996). Lenguaje e interacción en la educación escolar. Bogotá, Colombia: Universidad Distrital Francisco José de Caldas/Colciencias.

Linaza, J. \& Maldonado, A. (1987). Los juegos y el deporte en el desarrollo psicológico del niño. Barcelona, España: Anthropos.

Manrique, M. S. \& Rosemberg, C. R. (2009). El lenguaje infantil en situaciones de juego en el jardín de infantes. Summa Psicológica UST, 6(2), 105-118.

Michaels, S. (1988). Presentaciones narrativas: una preparación oral para la alfabetización con alumnos de primer curso. En J. Cook-Gumperz (Ed.), La construcción social de la alfabetización (pp. 109-135). Barcelona, España: Paidós.

Mondada, L. \& Pekarek Doehler, S. (2001). Interactions acquisitionnelles en contexte: Perspectives théoriques et enjeux didactiques [Interacciones adquisitivas en contexto: perspectivas teóricas y propuestas didácticas]. Le Français Dans le Monde, Numéro Spécial: Théories linguistiques et enseignement du français aux non-francophones(juillet), 107-142. 
Nelson, K. [Katherine] (1996). Language in cognitive development: Emergence of the mediated mind. New York, NY: Cambridge University Press.

Nelson, K. E. [Keith E.] (1977). Facilitating children's syntax acquisition. Developmental Psychology, 13, 101-107. doi:10.1037/0012-1649.13.2.101

Pekarek Doehler, S. (2006). Compétence et langage en action [Competencia y lenguaje en acción]. Bulletin Suisse de Linguistique Appliquée, 84, 9-45.

Pellegrini, A. D. (1982). The construction of cohesive text by preschoolers in two play contexts. Discourse Processes, 5, 101-108. doi:10.1080/01638538209544533

Pellegrini, A. D. (1985). Relations between preschool children's symbolic play and literate behavior. En L. Galda \& A. D. Pellegrini (Eds.), Play, language, and stories: The development of children's literate behavior (pp. 79-97). Norwood, NJ: Ablex.

Rosemberg, C. R. (2009). Las situaciones de juego: una matriz para el desarrollo infantil. En P. M. Sarlé (Coord.), Lo importante es jugar (pp. 41-56). Buenos Aires, Argentina: Homo Sapiens.

Rosemberg C. R. \& Borzone, A. M. (2004a). Programa Oscarcito: desarrollo lingüístico y cognitivo infantil. Manuscrito no publicado, Fundaciones Care (Alemania) y Árcor (Argentina), Buenos Aires, Argentina.

Rosemberg C. R. \& Borzone, A. M. (2004b). Proyecto de investigación "El desarrollo lingüístico y cognitivo en la primera infancia: un estudio psicolingüístico y sociocultural en barrios urbano marginales de Buenos Aires”. Manuscrito no publicado, Consejo Nacional de Investigaciones Científicas y Técnicas, Buenos Aires, Argentina.

Sacks, H., Schegloff, E. A. \& Jefferson, G. (1974). A simplest systematics for the organization of turn-taking for conversation. Language, 50, 696-735. doi:10.2307/412243

Sarlé, P. M. (2001). Juego y aprendizaje escolar: los rasgos del juego en la educación infantil. Buenos Aires, Argentina: Novedades Educativas.

Searle, J. R. (1986). Actos de habla. Madrid, España: Cátedra.

Seidman, S., Nelson, K. \& Gruendel, J. (1986). Make believe scripts: The transformation of ERs in fantasy. En K. Nelson (Ed.), Event knowledge: Structure and function in development (pp. 161-187). Hillsdale, NJ: Lawrence Erlbaum.

Silvestri, A. (1995). Discurso instruccional. Buenos Aires, Argentina: Universidad de Buenos Aires.

Simons, H. D. \& Murphy, S. (1988). Estrategias en el lenguaje hablado y en la aptitud de leer. En J. Cook-Gumperz (Ed.), La construcción social de la alfabetización (pp. 213-233). Madrid, España: Paidós.

Snow, C. E. (1983). Literacy and language: Relationships during the preschool years. Harvard Educational Review, 53, 165-189.

Snow, C. E., Porche, M. V., Tabors, P. O. \& Harris, S. R. (2007). Is literacy enough? Pathways to academic success for adolescents. Baltimore, MD: Paul H. Brookes.

Stein, A. \& Rosemberg, C. R. (2011). Aprender el sistema de escritura: un estudio de las modalidades de intervención de los adultos en situaciones de alfabetización familiar en poblaciones urbano-marginadas de Argentina. Summa Psicológica UST, 8(1), 47-62.

Stein, A. \& Rosemberg, C. R. (en prensa a). Redes de colaboración en situaciones de alfabetización familiar con niños pequeños: un estudio en poblaciones urbano marginadas de Argentina. Interdisciplinaria.

Stein, A. \& Rosemberg, C. R. (en prensa b). Alfabetización temprana en poblaciones urbano marginadas: la familia como contexto de oportunidades. Revista IRICE Nueva Época.

Stein, A. \& Rosemberg, C. R. (en prensa c). La lectura de cuentos en el hogar: matriz de oportunidades de aprendizaje para los niños. Cadernos de Educação.

Strauss, A. \& Corbin, J. (2002). Bases de la investigación cualitativa: técnicas y procedimientos para desarrollar la teoría fundamentada. Medellín, Colombia: Editorial Universidad de Antioquia.

Vigotsky, L. (1988). El desarrollo de los procesos psicológicos superiores. Barcelona, España: Grijalbo.

Watson, R. (1996). Talk about text: Literate discourse and metaliterate knowledge. En K. Reeder, J. Shapiro, R. Watson \& H. Goelman (Eds.), Literate apprenticeships: The emergence of language and literacy in the preschool years (pp. 81-100). Norwood, NJ: Ablex.

Fecha de recepción: Septiembre de 2010.

Fecha de aceptación: Marzo de 2012. 